Pacific Journal of Mathematics

ISOTOXXAL THINGS 


\title{
ISOTOXAL TILINGS
}

\author{
Branko Grünbaum and G. C. Shephard
}

\begin{abstract}
A plane tiling $\mathscr{T}$ is called isotoxal provided the group of symmetries of $\mathscr{T}$ acts transitively on the arcs (edges) of $\mathscr{T}$. A method for classifying isotoxal tilings is described, and the number of types of normal isotoxal tilings is determined.
\end{abstract}

1. A plane tiling $\mathscr{T}=\left\{T_{i} \mid i=1,2, \cdots\right\}$ is called isohedral if its symmetry group $S(\mathscr{T})$ is transitive on the tiles $T_{i}$ of $\mathscr{T}$, isogonal if $S(\mathscr{T})$ is transitive on the nodes (vertices) of $\mathscr{T}$, and isotoxal if $S(\mathscr{T})$ is transitive on the arcs (edges) of $\mathscr{T}$. (The word

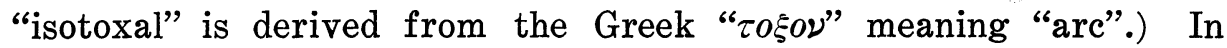
recent papers [2], [3] we have enumerated the different types of isohedral and isogonal tilings of the plane. Here we prove the following theorem.

THEOREM 1. There exist 26 types of normal isotoxal tilings of the plane. Of these, 25 are also either isohedral, or isogonal, or both.

By a "normal" tiling we mean one which is bounded (that is, the tiles are uniformly bounded in diameter and their inradii are uniformly bounded away from zero) and for which the intersection of any two tiles is either empty, or an edge, or a vertex of each. We thus exclude vertices of valence two, and digons. (As in [2] and [3], a vertex is any point common to at least three tiles, and a tile is called an $n$-gon if it has $n$ vertices and $n$ sides. Here $a$ side of a tile is the part of its boundary between consecutive vertices, and the word $n$-gon must not be taken to imply that the tile is convex or even that its sides are straight line segments.)

The proof of Theorem 1 will be given in the next section. It proceeds in two stages. First we determine the "combinatorial isotoxal tilings" and establish that there are exactly 30 types of these. Secondly we show that 26 of these types can be realized by actual tilings. Thus it will be seen that the procedure is analogous to that used in enumerating the isohedral and isogonal tilings, though the detailed analysis is rather different.

The third section will deal with tilings which are not normal. Here the main result will be the following.

THEOREM 2. There exist 15 types of bounded isotoxal tilings 
which contain digons, and a further 15 types which contain vertices of valence 2.

Theorem 2 can be proved in a similar way to Theorem 1, but it turns out to be simpler to proceed in a different manner. We shall show how all these additional tilings can be derived from the 30 types of combinatorial normal tilings by two processes, called edge-bifurcation and edge-division. Details will be given in $\S 3$.

The final section of the paper will be devoted to a brief explanation of the concept of duality as it applies to isotoxal tilings, and also to explaining some further relationships between isotoxal, isohedral and isogonal tilings.

Our main results are summarized in Table I. In Figures 4 and 6 we give diagrams of 41 types of isotoxal tilings, and in Figure 3 "marked" tilings that examplify the four types that cannot be realized using "unmarked" tiles. As will be explained later, the appearance of the remaining 15 types of tiling (those with vertices of valence 2) can be easily deduced from these.

As far as we are aware, the only previous attempt at enumerating isotoxal tilings is in an early paper of Heesch [5]. He correctly lists the various nets that occur, and he introduces, without explanation, a concept of "topologische Gleichwertigkeit" between vertices. In this way he arrives at a list of 28 isotoxal tilings. Because of certain obscurities in the treatment, direct comparison with our enumeration is impossible, but on any reasonable interpretation, Heesch's list is incomplete.

The analogous investigation of isotoxal tilings of the 2-dimensional sphere and the isotoxal convex polyhedra in 3-space has been carried out and will appear in [4].

2. The net $N(\mathscr{T})$ of a tiling $\mathscr{T}$ is defined as the graph consisting of the vertices and edges (arcs) of $\mathscr{T}$. We begin by determining the combinatorial types of net that can arise when $\mathscr{T}$ is normal and isotoxal.

Let $\alpha$ be an edge of $N(\mathscr{T})$ joining a vertex of valence $p$ to a vertex of valence $q$. The edge $\alpha$ must coincide with the sides of the two tiles which contain it, and we shall suppose that one of these is an $n$-gon and the other is an $m$-gon. Because $\mathscr{T}$ is normal and isotoxal, the application of Euler's theorem ([1], [6]) may be justified. From this it is easy to deduce the relation

$$
\frac{1}{n}+\frac{1}{m}+\frac{1}{p}+\frac{1}{q}=1 \text {. }
$$


We require solutions of this equation in integers with $m \geqq n \geqq 3$ and $q \geqq p \geqq 3$. However, there is a further restriction on the values, which arises in the following way. As we go round any tile, the vertices must be alternately $p$-valent and $q$-valent. Hence if $m$ or $n$ is odd, then $p$ and $q$ must be equal. Similarly, if $p$ or $q$ is odd then $m$ and $n$ must be equal. Integers satisfying these conditions are readily determined. In fact there are only five possibilities. If we use the notation $\langle n . m ; p . q\rangle$ and employ exponents to abbreviate where possible, the five solutions are

$$
\left\langle 3^{2} ; 6^{2}\right\rangle,\left\langle 3.6 ; 4^{2}\right\rangle,\left\langle 4^{2} ; 3.6\right\rangle,\left\langle 4^{2} ; 4^{2}\right\rangle \text { and }\left\langle 6^{2} ; 3^{2}\right\rangle \text {. }
$$

To see that five nets corresponding to these symbols actually exist, it is only necessary to remark that four are realized by the nets of uniform tilings and the fifth by a Laves net, see Figure 1. In fact, these nets are uniquely determined by the symbols.

Now let us suppose that we are given a normal isotoxal tiling $\mathscr{T}$. Let $T_{i}$ be any tile of $\mathscr{T}$ and assign a symbol, say $a$, to any directed (oriented) side of $T_{i}$. Applying the operations of the symmetry group $S(\mathscr{T})$ will yield a corresponding assignment of this symbol $a$ to certain sides of other tiles of $\mathscr{T}$. Not only may two or more sides of the same tile be assigned the symbol $a$, but it may also happen that this same symbol is assigned a second time to the same side of $T_{i}$ but in a reversed direction. In this case we regard $a$ as a label for an undirected (unoriented) side of $T_{i}$.

Each edge $\alpha$ of $N(\mathscr{T})$ is a side of two tiles, and the fact that $S(\mathscr{T})$ is transitive on these edges implies that at least one of the two sides of tiles that coincide with $\alpha$ must be labelled $a$. If both sides bear the symbol $a$, then there is nothing further to be done. If not, we label the other directed side of a tile at $\alpha$ with the symbol $b$ and apply the operations of $S(\mathscr{T})$ in an exactly similar manner. In this way, all the sides are labelled.

To define the edge symbol of $\mathscr{T}$ we first write down the symbols assigned to the sides of the two tiles at $\alpha$ (either $a a$ or $a b$ ) and then add superscripts ${ }^{+},-$or neither, to these symbols, as follows:

(i) If the two sides are oriented in opposite directions, then the superscripts are ${ }^{++}$.

(ii) If the two sides are oriented in the same direction, then the superscripts are ${ }^{+-}$.

(iii) If the two sides are unoriented, then no superscripts are used.

Hence, ignoring trivial changes of notation, there are just five possible edge symbols, namely $a^{+} b^{+}, a^{+} a^{+}, a^{+} a^{-}, a b$ and $a a$. It will 


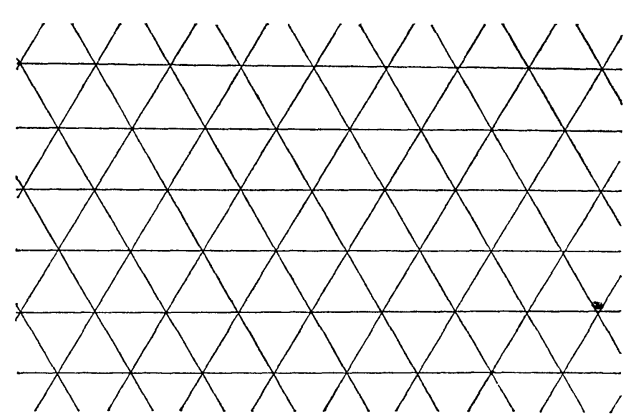

$\left\langle 3^{2} ; 6^{2}\right\rangle$

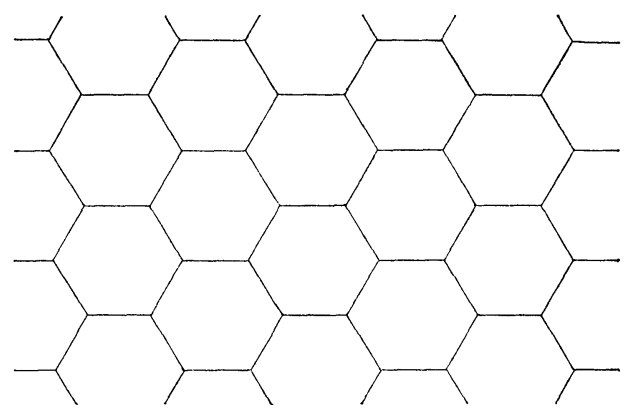

$\left\langle 6^{2} ; 3^{2}\right\rangle$

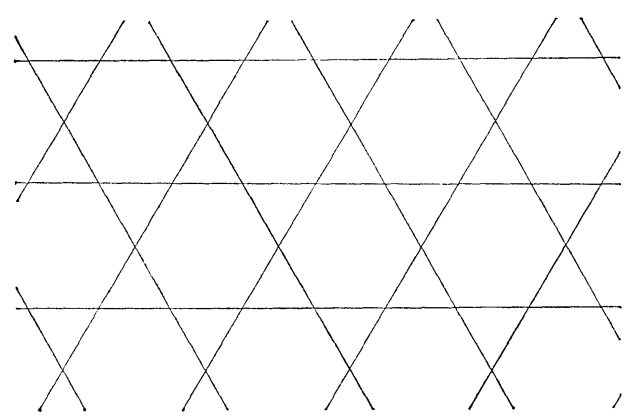

$\left\langle 3.6 ; 4^{2}\right\rangle$

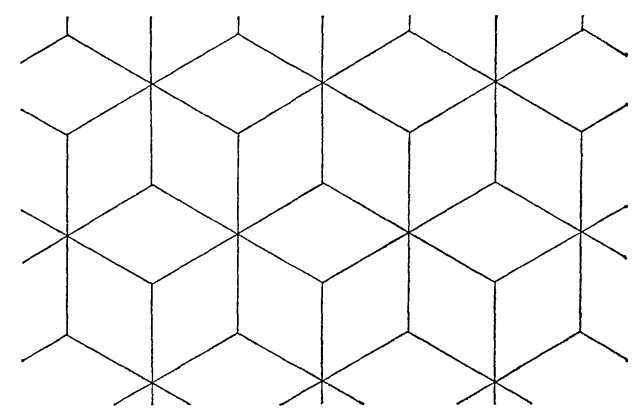

$\left\langle 4^{2} ; 3.6\right\rangle$

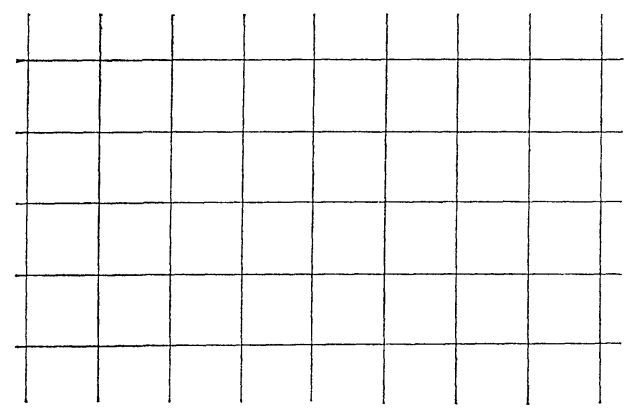

$\left\langle 4^{2} ; 4^{2}\right\rangle$

FIgURE 1. The five isotoxal nets. 
be seen that, in effect, the tile symbol is obtained by reading off the symbols that occur as we proceed cyclically round an edge and the superscripts indicate the relative orientations of the sides of the tiles. In a similar manner the adjacency symbol of $\mathscr{T}$ is defined. Here we proceed cyclically round the two tiles of which a side coincides with $\alpha$, reading off the symbols in order. We use superscripts,+- , or neither, to indicate whether the symbol relates to a side which is oriented coherently, or oppositely, or is undirected. If the two tiles which abut on $\alpha$ have identical symbols, then we need state only one of them; otherwise both must be given in the adjacency symbol.

Examples of edge and adjacency symbols appear in Figure 2. The first of these has net $\left\langle 4^{2} ; 4^{2}\right\rangle$ which is the net of the regular tiling by squares. The edge symbol is $a^{+} b^{+}$and the adjacency symbol is $a^{+} b^{+} b^{-} a^{-}$. The second has net $\left\langle 3.6 ; 4^{2}\right\rangle$ which is the net of

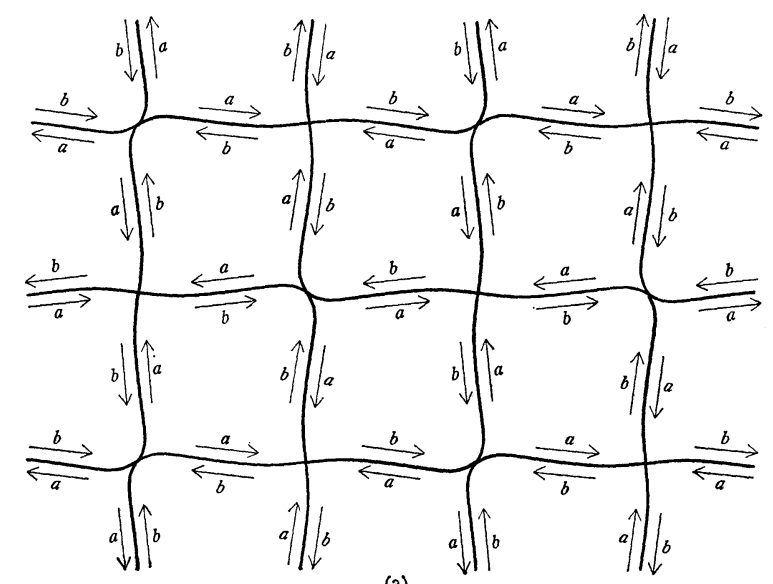

(a)

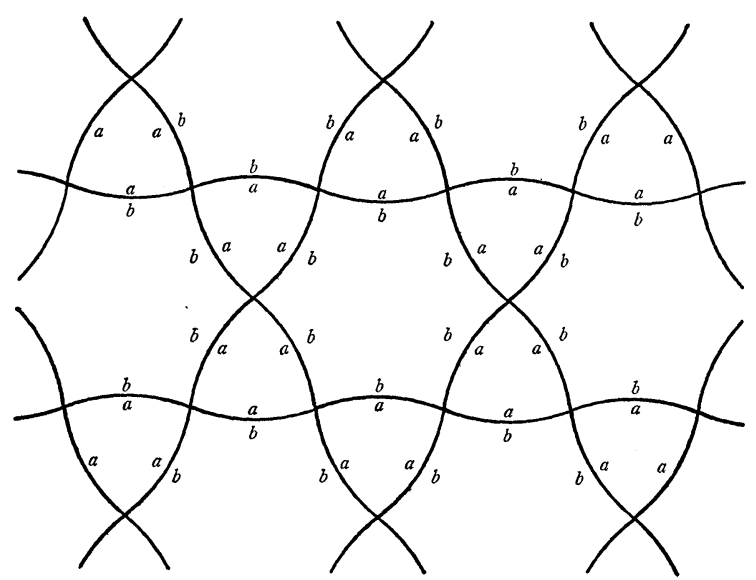

(b)

FigURE 2. 
the uniform tiling by triangles and hexagons, two of each meeting at each vertex. Here the edge symbol is $a b$ and the adjacency symbol is $a a a ; b b b b b b$.

DEFINITION. Two isotoxal tilings are of the same type if they have the same edge and adjacency symbols.

Of course we do not consider edge or adjacency symbols as distinct if they differ trivially, that is, by change of notation or by permutation.

The first stage in the enumeration of the normal isotoxal tilings is the determination of the number of combinatorial types. To do this, we take in turn each of the five possible nets, and assign to it edge and adjacency symbols in all possible ways. The number of possibilities is extremely limited. There are only five different edge symbols, and the edges of a triangular tile can be labelled in only two essentially different ways: $a^{+} a^{+} a^{+}$or $a a a$. In the case of hexagonal tiles there are five possible labellings and for square tiles there are nine. But many of these can be eliminated immediately since they are not combinatorially possible. By this we mean that we cannot label all the edges and all the tiles of the chosen net in a manner consistent with the edge and adjacency symbols under consideration.

We finally arrive at a list of 30 labellings, and these are given in Table I. They do not correspond to isotoxal tilings as originally defined, but to "combinatorial types" of tilings, since they depend essentially on the labelling of the edges. A convenient way to display these types is by means of marked tilings. Examples are given in Figure 3 of marked tilings of types IT 3, IT 21, IT 22, and IT 28 of the table. To construct a marked tiling we take a realization of the net as in Figure 1 (with either regular or congruent tiles), and then assign a mark to one tile of each transitivity class. The mark may be chosen arbitrarily so long as its symmetry group is the trivial group. We have selected $L$ in each case. The operations of $S(\mathscr{T})$ yield the corresponding markings of all the other tiles. Each tile will carry more than one mark, which will thus indicate the group of symmetries of the tile induced by $S(\mathscr{T})$. In Figure 3 these induced groups are $C_{3}, C_{4}, D_{2}$, and $D_{3}$, respectively.

Our final task is to see which of these thirty combinatorial types of tilings can be represented by actual tilings. That is to say, we must determine whether the shape of the tiles can be chosen so that the resulting tiling is of the required type. The technique for doing so can be described as follows. Firstly we draw the net with either regular or congruent tiles and straight edges of equal lengths, as in Figure 1. Secondly, we replace the edges of the net in the 


\begin{tabular}{|c|c|c|c|c|c|c|c|c|c|c|c|c|c|c|c|c|}
\hline 离: & 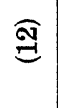 & ๓ึ & लె & ஜ్ & ผొ & ஸ్ & $\vec{m}$ & ణి & ๙ิ & $\vec{\omega}$ & లి & ๙ั & นด & น้น & $\stackrel{\infty}{\rightarrow}$ & ำ \\
\hline 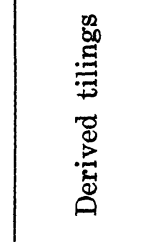 & $\widehat{\Xi}$ & & $\begin{array}{l}\text { ลี } \\
\text { E- } \\
\text { สิ } \\
\text { E- }\end{array}$ & $\begin{array}{l}\text { ले } \\
\text { E }\end{array}$ & $\begin{array}{l}\text { D } \\
E\end{array}$ & 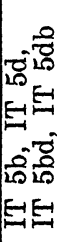 & & & $\begin{array}{l}\not \\
\infty \\
E\end{array}$ & & & $\begin{array}{l}\stackrel{0}{二} \\
\underline{G}\end{array}$ & & & & \\
\hline 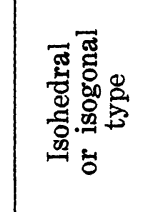 & 응 & $\begin{array}{l}\text { 움 } \\
\text { 乌 }\end{array}$ & 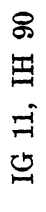 & 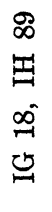 & $\begin{array}{l}\stackrel{9}{9} \\
\underset{=}{⿹}\end{array}$ & 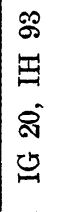 & 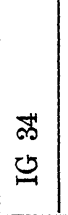 & $\begin{array}{l}\infty \\
\infty \\
0 \\
0\end{array}$ & 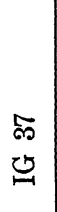 & $\begin{array}{l}\text { ल゙ } \\
\text { 龱 }\end{array}$ & $\begin{array}{l}\text { e्r } \\
\text { 龱 }\end{array}$ & $\begin{array}{l}5 \\
5\end{array}$ & $\begin{array}{l}-1 \\
0 \\
0\end{array}$ & $\begin{array}{l}E \\
\vdots\end{array}$ & & $\begin{array}{l}5 \\
6\end{array}$ \\
\hline $\begin{array}{l}\overrightarrow{\widetilde{I}} \\
\overrightarrow{\tilde{\theta}}\end{array}$ & $\widehat{\theta}$ & 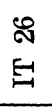 & 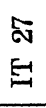 & $\begin{array}{l}\text { S } \\
\text { E }\end{array}$ & 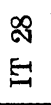 & $\begin{array}{l}\text { ติ } \\
E\end{array}$ & $\begin{array}{l}\infty \\
E\end{array}$ & $\begin{array}{l}\text { 울 } \\
\text { ㅌ-1ㅂ }\end{array}$ & $\begin{array}{l}\not 1 \\
\xi\end{array}$ & $\begin{array}{l}0 \\
\xi\end{array}$ & 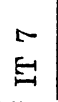 & $\begin{array}{l}\infty \\
\xi\end{array}$ & $\begin{array}{l}\stackrel{20}{G} \\
\qquad\end{array}$ & 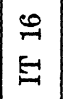 & $\begin{array}{l}\underset{H}{H} \\
E \\
*\end{array}$ & $\begin{array}{l}\stackrel{N}{\sim} \\
\stackrel{5}{E}\end{array}$ \\
\hline 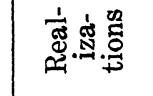 & $\widehat{\infty}$ & z & z & $\Sigma$ & Z & ט & ' & z & 0 & z & z & 0 & z & z & z & z \\
\hline 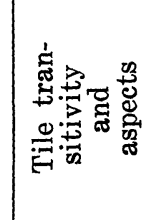 & $\Xi$ & 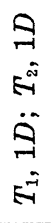 & ה & $\begin{array}{l}\text { 胥 } \\
\text { ă } \\
\text { Fi }\end{array}$ & $\begin{array}{l}\ddot{H} \\
\stackrel{-}{*} \\
\ddot{H} \\
\ddot{H}\end{array}$ & $\begin{array}{l}\text { o } \\
\text { Fi }\end{array}$ & $\begin{array}{l}\vec{\theta} \\
\ddot{2} \\
\ddot{\theta} \\
\dot{\theta}\end{array}$ & 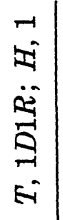 & $\begin{array}{l}\vec{y} \\
\ddot{A} \\
\ddot{A}\end{array}$ & ఠి & $\stackrel{\infty}{0}$ & $\infty$ & $\begin{array}{c}\vec{z} \\
\dot{\sigma} \\
\ddot{\theta} \\
\dot{\vec{\sigma}} \\
\dot{\sigma}\end{array}$ & $\begin{array}{c}v \\
\tilde{\sigma} \\
\ddot{\theta} \\
\dot{\sigma}\end{array}$ & $\begin{array}{c}\ddot{1} \\
\dot{\sigma} \\
\ddot{\ddot{\sigma}} \\
\dot{\sigma}\end{array}$ & $\begin{array}{l}\text { बे } \\
0\end{array}$ \\
\hline 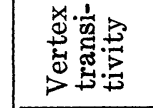 & $\widehat{\theta}$ & ఫ్ర & ఫ్ & ఫ & ఫ్ర & $\check{\mho}$ & $\check{8}$ & ષ્ & ఫ్ర & $\frac{\alpha}{\gamma}$ & $\frac{\infty}{\gamma}$ & $\frac{\infty}{\gamma}$ & $\Xi$ & ఫ్ & $\frac{\infty}{\gamma}$ & $\frac{\infty}{\gamma}$ \\
\hline 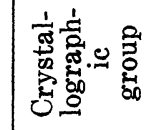 & $\widehat{20}$ & $\stackrel{2}{2}$ & $\stackrel{2}{\circ}$ & 祢 & 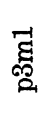 & દ్ష & 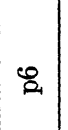 & 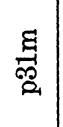 & ๕్વ & 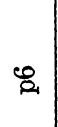 & 䄈 & \&્ّ & 范 & $\frac{100}{20}$ & घี & 范 \\
\hline 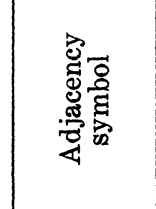 & $\underset{J}{ت}$ & $\begin{array}{l}+ \\
\pm \\
+ \\
+ \\
0 \\
0 \\
+ \\
0 \\
+ \\
0 \\
0 \\
0\end{array}$ & $\begin{array}{l}+ \\
+ \\
+ \\
+ \\
+ \\
0\end{array}$ & $\begin{array}{l}+ \\
0 \\
+ \\
+ \\
+ \\
0\end{array}$ & $\begin{array}{l}8 \\
0 \\
0 \\
8\end{array}$ & $\underset{ఫ}{8}$ & 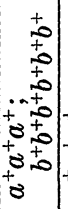 & $\begin{aligned} & 1 \\
& \pm \\
& 1 \\
& 1 \\
& \cdots \\
& \cdots \\
& 0 \\
& 0 \\
& 0 \\
& 0 \\
& 0 \\
& 0 \\
& 0 \\
& 0\end{aligned}$ & $\begin{array}{l}0 \\
0 \\
00 \\
0 \\
08 \\
8\end{array}$ & $\begin{array}{l}+ \\
0 \\
+ \\
0 \\
+ \\
+ \\
0\end{array}$ & $\begin{array}{l}1 \\
0 \\
0 \\
0 \\
+ \\
0 \\
0 \\
0\end{array}$ & $\begin{array}{l}1 \\
0 \\
+0 \\
0 \\
1 \\
0 \\
0 \\
0\end{array}$ & 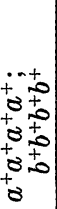 & $\begin{array}{lll}0 & 1 \\
0 & 1 \\
0 & 7 \\
0 & 0 \\
0 & 0 \\
0 & 0 \\
0 & 7 \\
0 & 0 \\
0 & 0\end{array}$ & $\left|\begin{array}{lll}0 & 1 \\
0 & 0 \\
0 & 7 \\
+ & 0 \\
0 & 0 \\
0 & 0 \\
0 & 0 \\
0 & 0 \\
0 & 0\end{array}\right|$ & $\begin{array}{l}+ \\
+ \\
+ \\
0 \\
+ \\
+0 \\
+0\end{array}$ \\
\hline 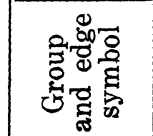 & $\widehat{\infty}$ & $\begin{array}{l}+ \\
+ \\
+ \\
0 \\
01\end{array}$ & $\begin{array}{l}+ \\
+ \\
+ \\
0 \\
0\end{array}$ & $\begin{array}{l}1 \\
0 \\
0 \\
0 \\
0\end{array}$ & $\begin{array}{l}8 \\
8\end{array}$ & $\underset{8}{8}$ & $\begin{array}{l}+ \\
+ \\
0 \\
0 \\
\text { ti }\end{array}$ & & $\begin{array}{l}8 \\
8 \\
8\end{array}$ & 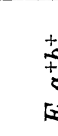 & & $\begin{array}{l}1 \\
0 \\
0 \\
0 \\
\tilde{A}\end{array}$ & & & & \\
\hline $\begin{array}{l}\overrightarrow{0} \\
\ddot{z}\end{array}$ & $\widehat{\overparen{U}}$ & & & $\begin{array}{l}\hat{\Xi} \\
\ddot{\sigma}\end{array}$ & & & & $\begin{array}{l}\widehat{\hat{\psi}} \\
\ddot{\theta} \\
\ddot{\theta}\end{array}$ & & & $\begin{array}{l}\hat{0} \\
\text { in } \\
\ddot{0} \\
\dot{\Xi}\end{array}$ & & & $\sum^{\mathbb{N}}$ & & \\
\hline 苔薝 & $\cong$ & $\vec{E}$ & $\stackrel{N}{E}$ & 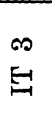 & $\underset{⿱ ㇒}{E}$ & $\begin{array}{l}20 \\
E\end{array}$ & $\begin{array}{l}\infty \\
E\end{array}$ & $E$ & $\begin{array}{l}\infty \\
\Theta\end{array}$ & E & 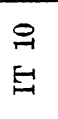 & $\begin{array}{l}F \\
F \\
E\end{array}$ & $\underset{\mathcal{F}}{\mathcal{E}}$ & $\stackrel{\oplus}{\stackrel{\oplus}{-}}$ & $\begin{array}{l}ت \\
E \\
E\end{array}$ & $\begin{array}{l}\text { 瓷 } \\
\text { E- }\end{array}$ \\
\hline
\end{tabular}




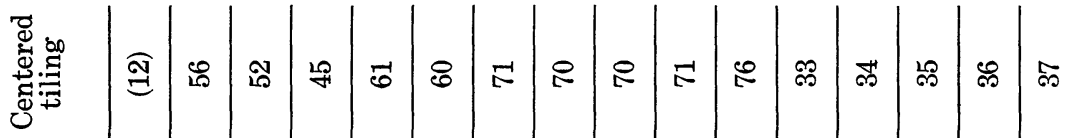


\begin{tabular}{|c|c|c|c|c|c|c|c|c|c|c|c|c|c|c|c|c|}
\hline 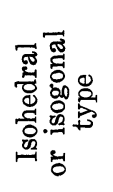 & 을 & E & 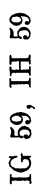 & 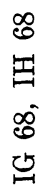 & 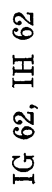 & 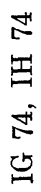 & 兒 & $\begin{array}{l}\stackrel{2}{2} \\
\text { 四 }\end{array}$ & 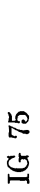 & 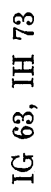 & 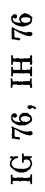 & $\begin{array}{l}\text { 음 } \\
\text { 四 }\end{array}$ & 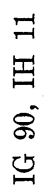 & $\begin{array}{l}\stackrel{\sigma}{\sim} \\
\text { II }\end{array}$ & 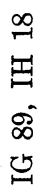 & 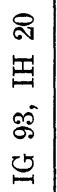 \\
\hline
\end{tabular}

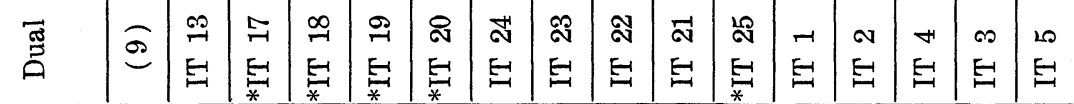

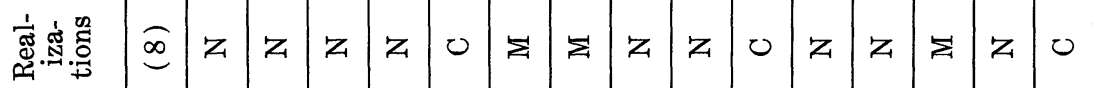

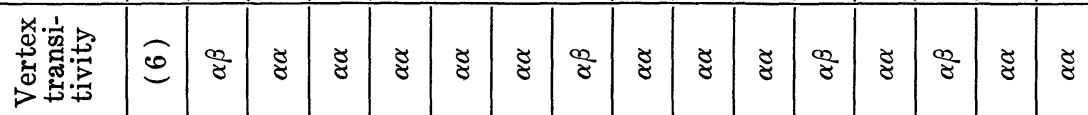

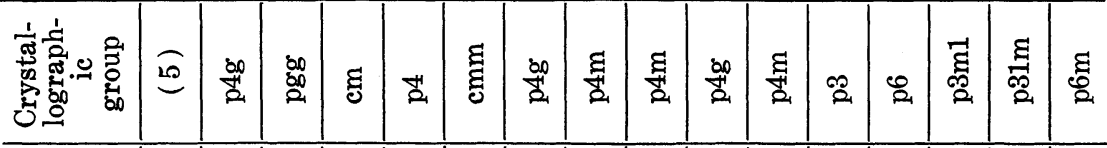

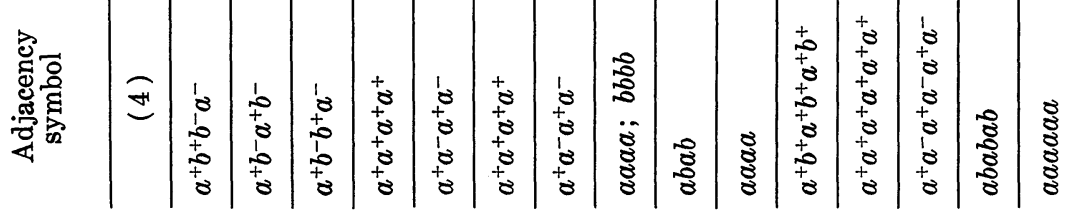

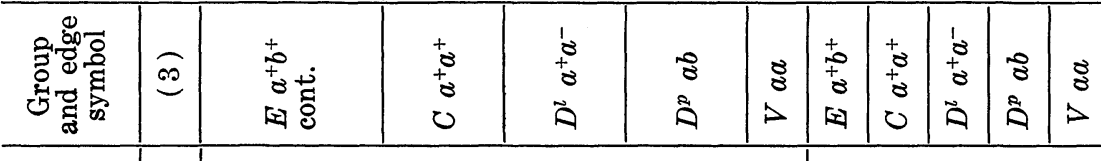
苋 范 สิ हैं

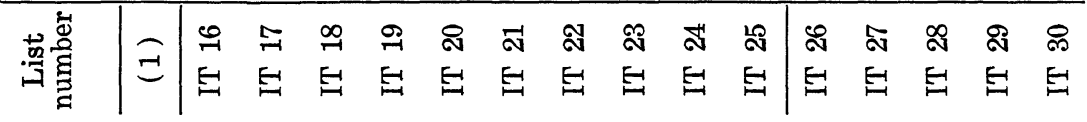


Note to Table I: Column (1) gives the list number, and column (2) indicates the net of the tiling in the notation of Figure 1. Column (3) gives the possible edge symbols and edge groups (that is, the restriction of $S(\mathscr{T})$ to the neighborhood of one edge), and column (4) shows all possible adjacency symbols. The notation for the edge and adjacency symbols follows that of $\S 2$ and the groups are designated as follows: $E$ is the trivial group, $C$ is the cyclic group of order 2 containing reflection in the midpoint of the edge, $D^{l}$ and $D^{p}$ are dihedral groups of order 2 containing reflections in lines along, and perpendicular to, the edge, respectively, and $V$ is the 4-group containing $C$, $D^{l}$ and $D^{p}$ as subgroups.

Column (5) shows the crystallographic group of the tiling using the international crystallographic notation (as in [2] and [3]). Column (6) indicates the transitivity classes of the vertices in a similar notation to that of [2] and column (7) indicates both the transitivity classes of tiles and also their aspects in the notation of [3]. In column (8) we show possible realizations of the tiling with the following abbreviations:

$\mathrm{C}$ : Isotoxal tiling by convex polygons exists.

$\mathrm{N}$ : Isotoxal tilings exist, but not with convex tiles.

M: A marked isotoxal tiling exists, but the type cannot be realized by an unmarked isotoxal tiling.

Column (9) indicates the dual type of tiling as defined in $\S 4$, column (10) gives the list number of the tiling in the tables of [2] or [3] if it is isohedral or isogonal, and column (11) indicates when derived tilings exist in the notation of $\S 3$. An entry $n$ in the column (12) means that the associated tile-centered and edge-centered tilings (as defined in $\S 4$ ) are of types $\mathrm{IH} n$ and $\mathrm{IG} n$, respectively.

following way:

(i) If the edge symbol is $a^{+} a^{+}$we replace each edge by a $C$ curve, that is, any curve which has the midpoint of the edge as a center of symmetry.

(ii) If the edge symbol is $a b$ we replace each edge by a $D$ curve, that is one for which the perpendicular bisector of the edge is a line of reflective symmetry.

(iii) If the edge symbol is $a^{+} b^{+}$then we replace the edge by an $E$-curve, that is, any unsymmetric arc.

In the case of the other edge symbols $\left(a^{+} a^{-}\right.$or $\left.a a\right)$ the edge must be left as a straight line segment. Thirdly we distort the net, but not the individual edges, if possible so that the crystallographic group of the tiling is unchanged. In each operation the only restriction is that the various curves chosen as edges, and the distortion of the third stage, must be such that the edges in the resulting tiling are disjoint except possibly at their endpoints. The final distortion in required only if we aim to find the most general tiling of the given type. For example, in the case of tiling IT 14, the squares of the original net may be distorted into rhombs since this does not alter the group $S(\mathscr{T})$, which in this case is pmm.

Eventually we find that of the 30 combinatorial types listed in Table I, exactly 26 types can be realized by normal isotoxal tilings, thus establishing the first statement of Theorem 1. Diagrams of the 26 types appear in Figure 4. The four types that cannot be so 


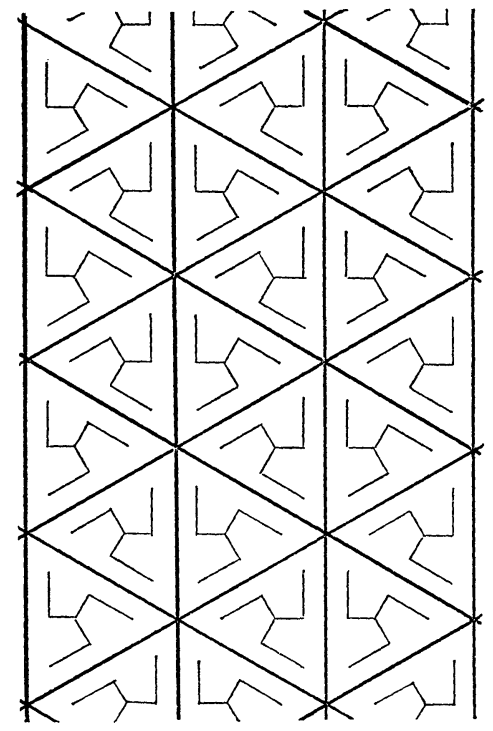

IT 3

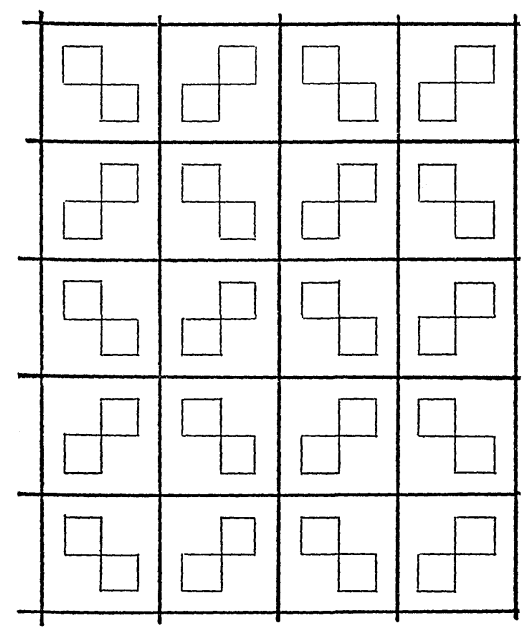

IT 22

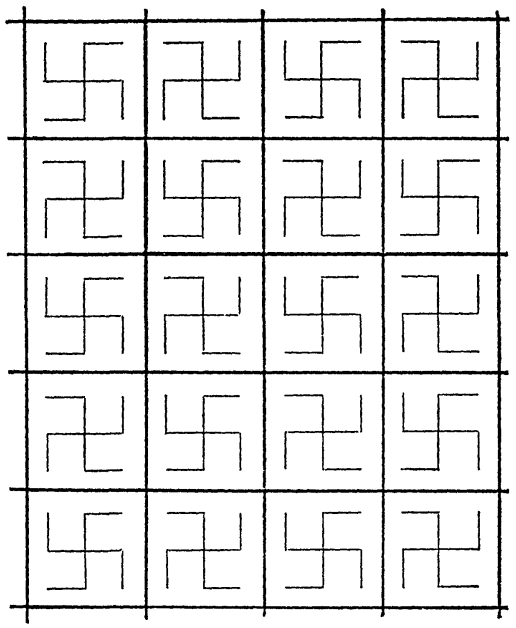

IT 21

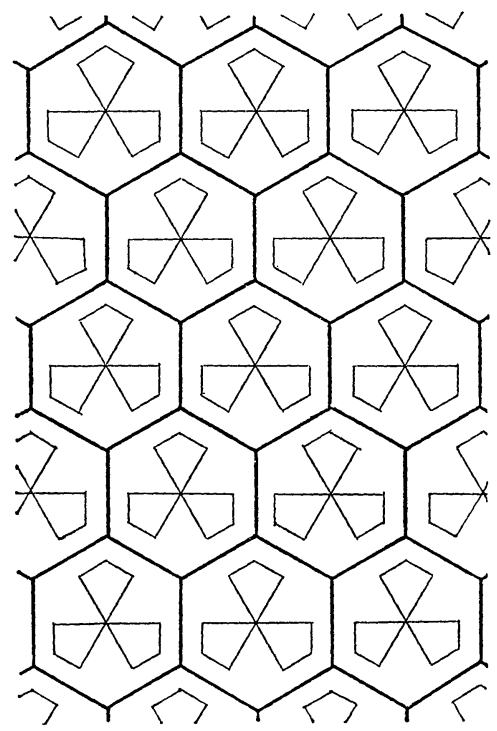

IT 28

FIgURE 3.

represented are IT 3 , IT 21 , IT 22 , and IT 28 . In each case the edge symbol is $a^{+} a^{-}$and so, in the second stage described above, the edges must be left as straight line segments. But then the tilings have additional symmetries (the edge symbols become $a a$ ) and so they are automatically of types IT 5, IT 25, IT 25 and IT 30 respectively. The diagrams of Figure 3 show the four types that can only be represented as marked tilings.

The final statement of the theorem follows by comparing the 


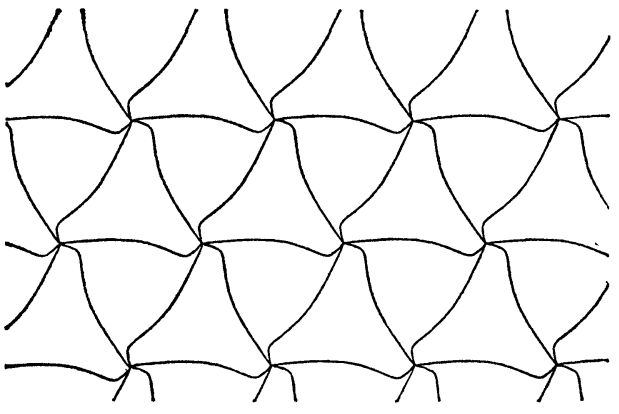

IT 1

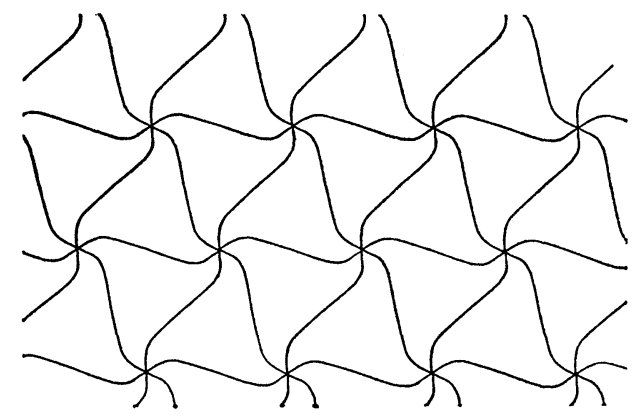

IT 2

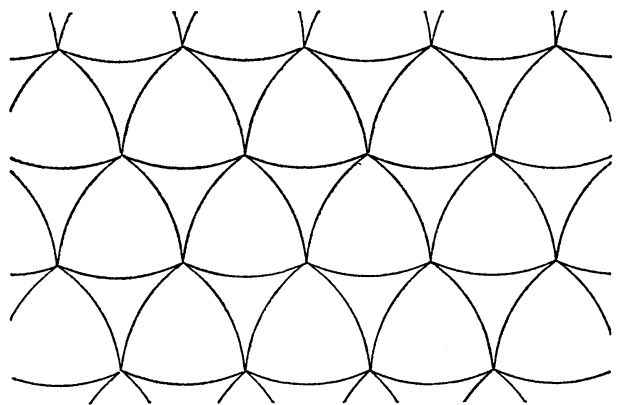

IT 4

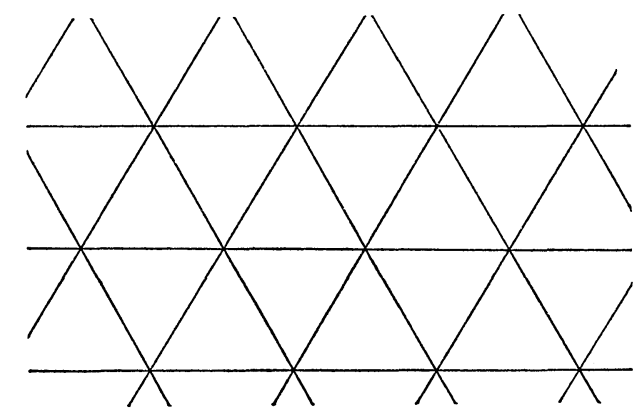

IT 5

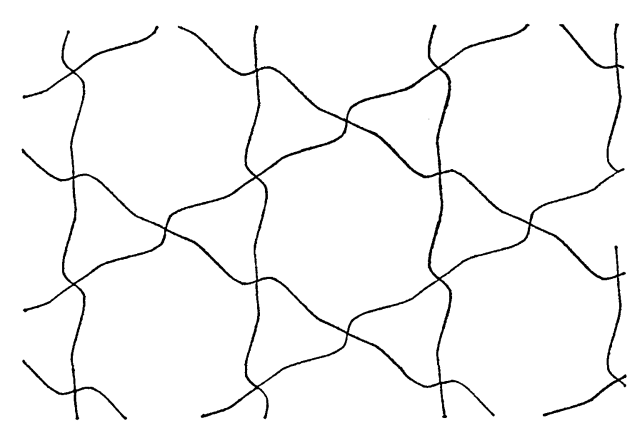

IT 6

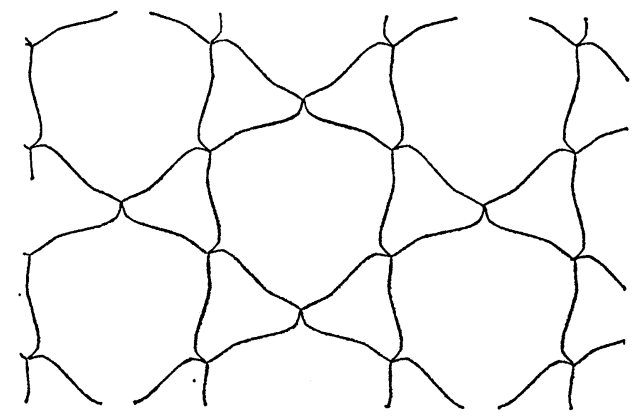

IT 7

Figure 4. The 26 types of isotoxal tilings. 
418

BRANKO GRUNBAUM AND G. C. SHEPHARD

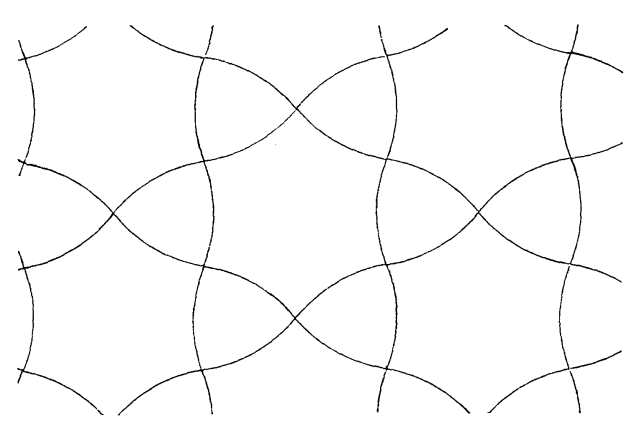

IT 8

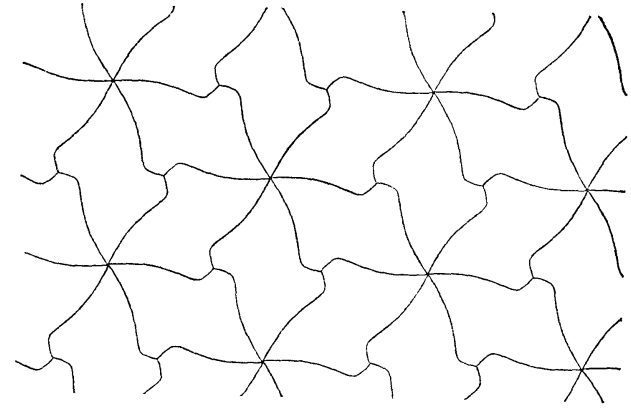

IT 9

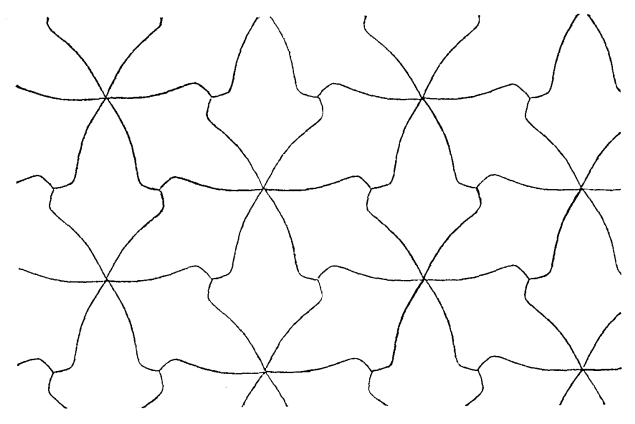

IT 10

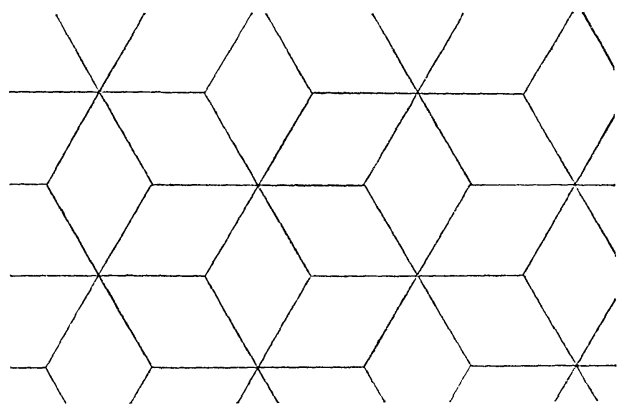

IT 11

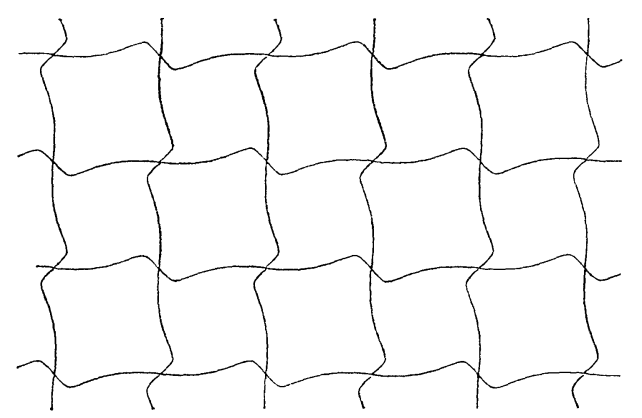

IT 12

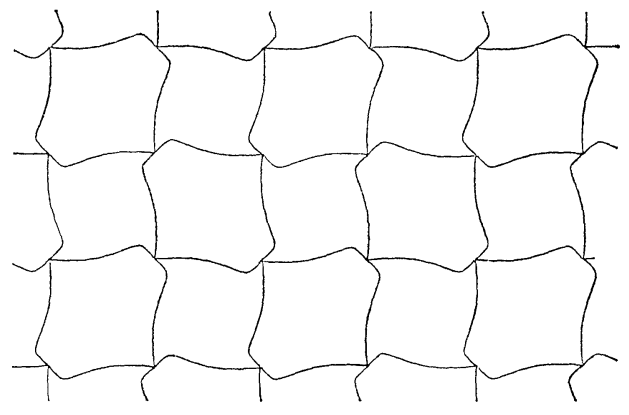

IT 13

FIgUre 4 (continued). 
ISOTOXAL TILINGS

419
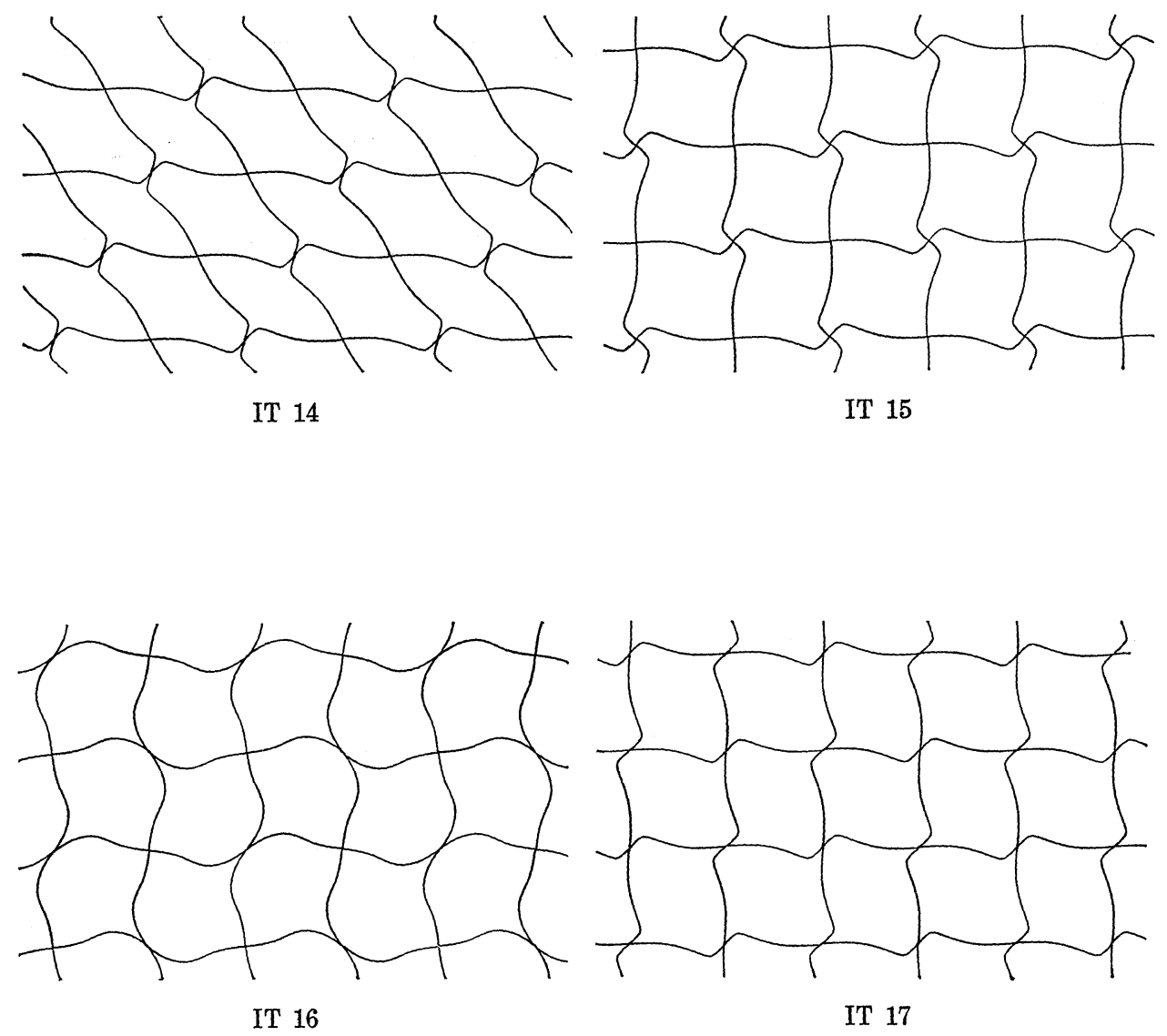

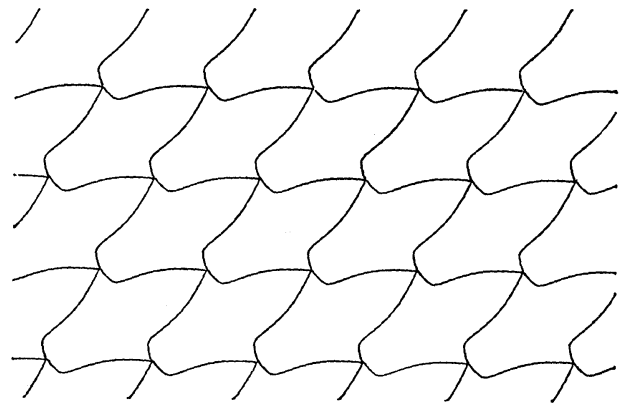

IT 18

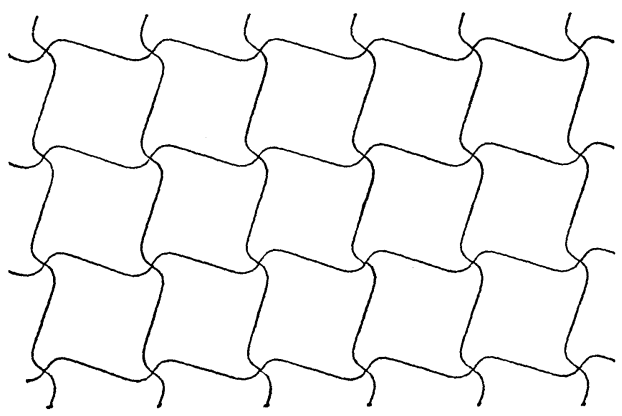

IT 19

Figure 4 (continued). 


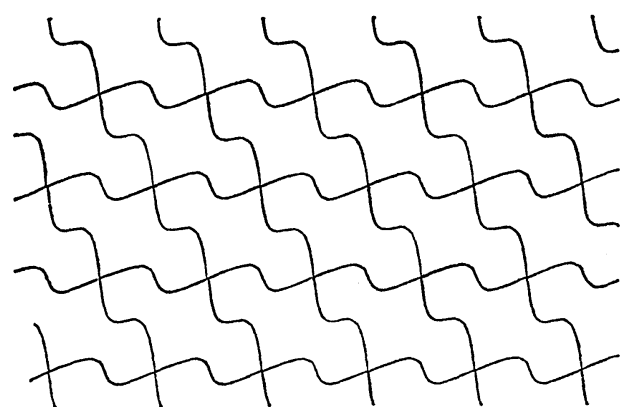

IT 20

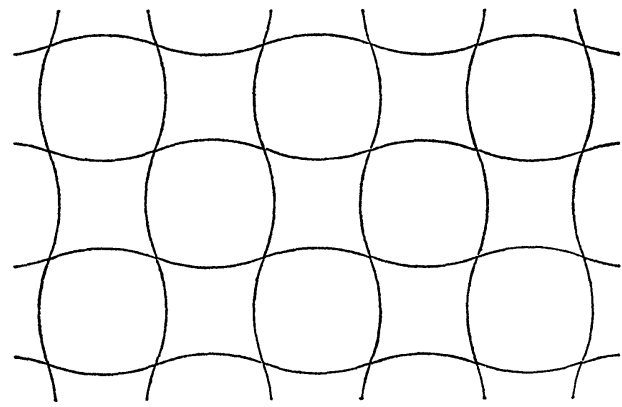

IT 23

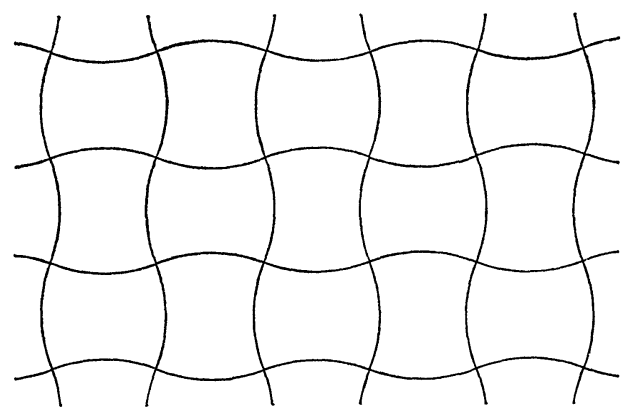

IT 24

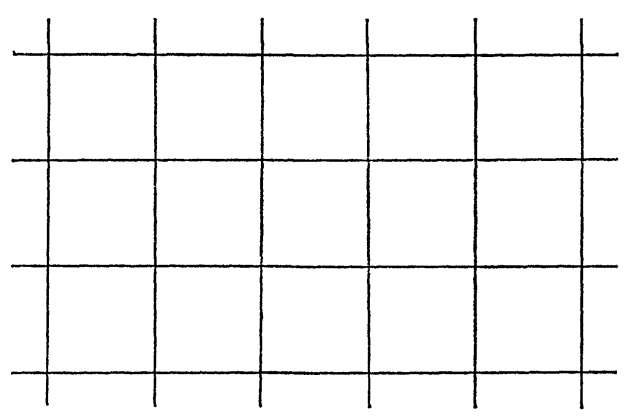

IT 25

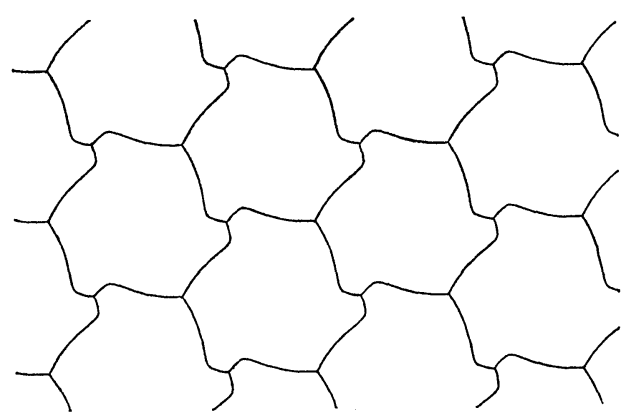

IT 26

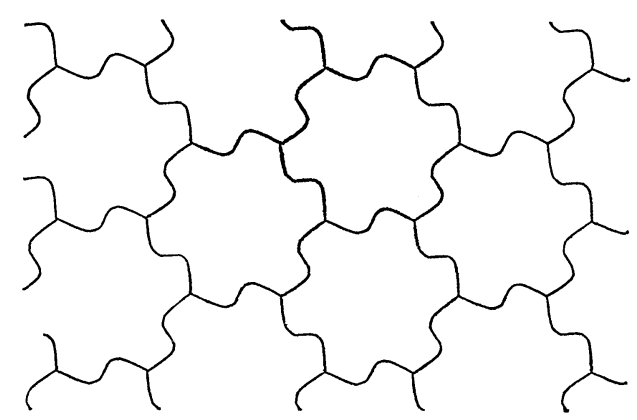

IT 27

Figure 4 (continued). 


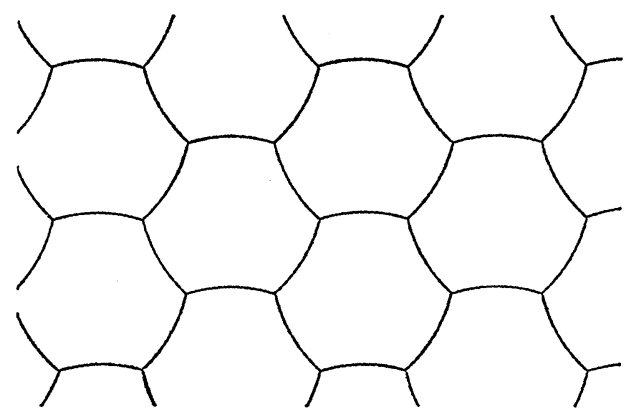

IT 29

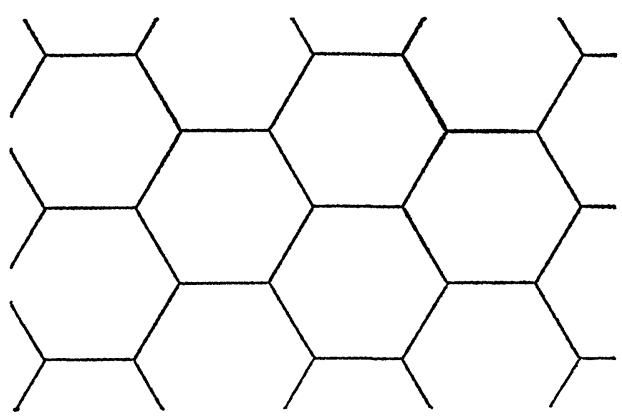

IT 30

Figure 4 (end).

tilings we have found with those in the tables of [2] and [3]. Twenty-five types turn out to be isohedral, isogonal, or both, and appropriate references are given in column (11) of Table I. In this way the proof of Theorem 1 is completed.

In particular, we draw attention to tiling IT 14 . This is the only normal isotoxal tiling which is neither isohedral nor isogonal. On the other hand, in addition to the three regular tilings there exist eight types (IT 2, IT 17, IT 18, IT 19, IT 20, IT 24, IT 27, IT 29) which are isohedral, isogonal and isotoxal. Two types (IT 3, IT 21) of marked tilings also have all three properties.

3. We now consider bounded isotoxal tilings that include tiles which are digons or have vertices of valence two. To find the corresponding nets we proceed as described at the beginning of the preceding section. As before we find symbols $\langle n . m ; p . q\rangle$ satisfying the same conditions except that here we allow the possibility that one of $n, p$ may have the value 2 . In this way we arrive at a list of fourteen additional nets, namely:

$$
\begin{aligned}
& \left\langle 2.3 ; 12^{2}\right\rangle,\langle 2.4 ; 6.12\rangle,\left\langle 2.4 ; 8^{2}\right\rangle,\langle 2.6 ; 4.12\rangle, \\
& \left\langle 2.6 ; 6^{2}\right\rangle,\langle 2.8 ; 4.8\rangle,\langle 2.12 ; 4.6\rangle,
\end{aligned}
$$

and

$$
\begin{aligned}
& \left\langle 12^{2} ; 2.3\right\rangle,\langle 6.12 ; 2.4\rangle,\left\langle 8^{2} ; 2.4\right\rangle,\langle 4.12 ; 2.6\rangle, \\
& \left\langle 6^{2} ; 2.6\right\rangle,\langle 4.8 ; 2.8\rangle,\langle 4.6 ; 2.12\rangle .
\end{aligned}
$$

The seven nets of the first group are illustrated by tilings IT $5 \mathrm{~b}$, IT $11 \mathrm{~b}$, IT $25 \mathrm{~b}$, IT $5 \mathrm{db}$, IT $30 \mathrm{~b}$, IT $25 \mathrm{db}$, IT $30 \mathrm{db}$ in Figure 6 .

It is possible to assign edge and adjacency symbols to these as previously described, and so determine the combinatorial types of tilings. This will lead to a proof of Theorem 2, but it is simpler to proceed as follows. 
We observe that each of the above 14 nets can be derived from the 5 "normal" nets previously determined by applying one or two of the following operations:

(a) Edge-bifurcation. By this we mean that each edge is replaced by a digon. If the vertices at the ends of the original edge are $A, B$, then the vertices of the digon in the new tiling are $A$ and $B$.

(b) Edge-division. Here an edge is replaced by two edges meeting at a vertex of valence 2 . If the vertices at the ends of the original edge are $A, B$, then the ends of the new edges are $A, C$ and $C, B$, where $C$ is the new vertex of valence 2 .

Not only may these two operations be applied to the nets of isotoxal tilings but they may also be applied, under certain circumstances, to tilings with labelled edges. The method of doing this is illustrated in Figure 5. If the edge symbol is $a^{+} a^{+}, a^{+} a^{-}$or $a a$, then the edge-bifurcation yields a new type with edge symbol $a^{+} b^{+}$, $a^{+} b^{+}$or $a b$ respectively. The new adjacency symbol is obtained by adjoining $b^{+} b^{+}, b^{+} b^{-}$or $b b$ to that of the original tiling. If the edge symbol is $a^{+} a^{+}, a b$ or $a a$ then edge-division may be applied in an analogous manner. To other types of labelled edges the operations may not be applied.

Thus, for example, starting with tiling IT 30 we can obtain a

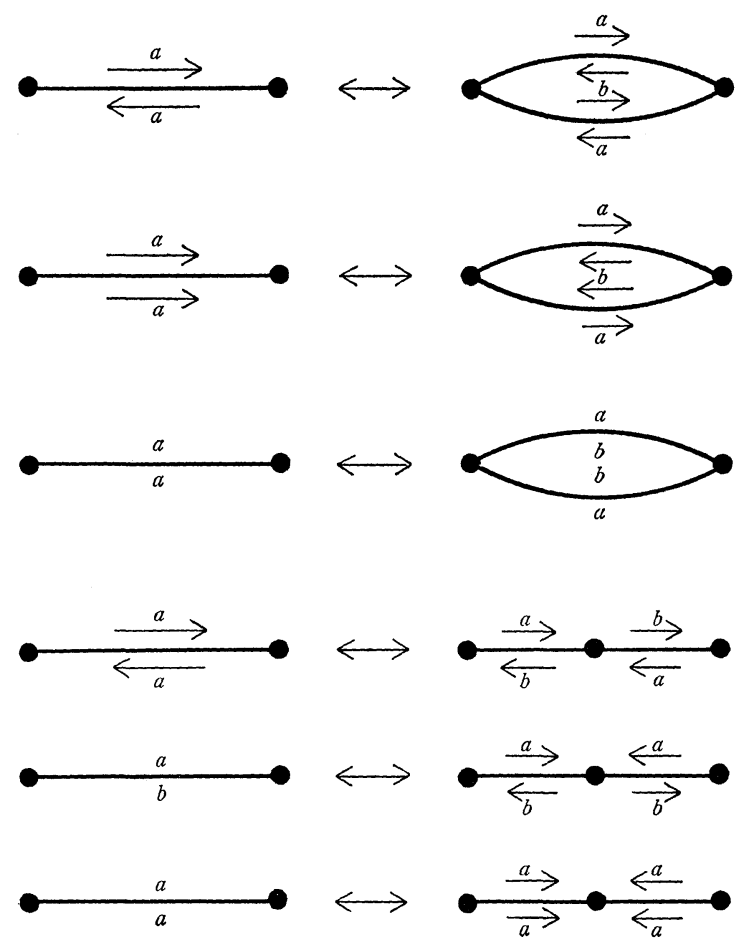

Figure 5. Arc-bifurcation and arc-division of labelled edges. 


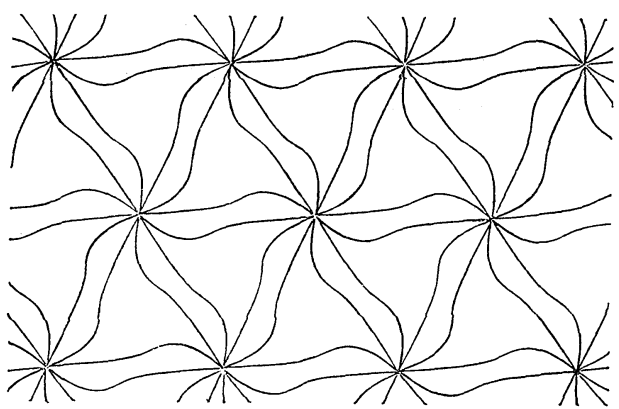

IT $2 b$

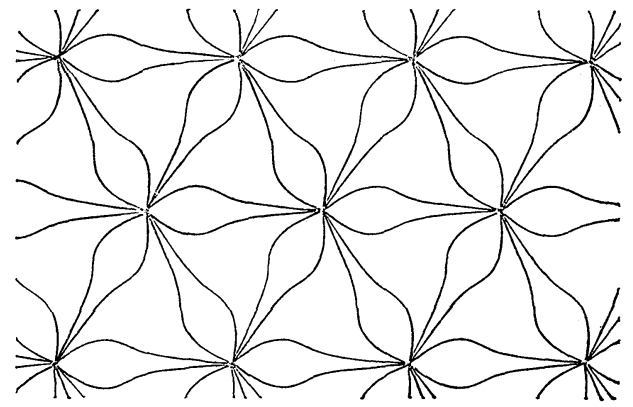

IT $3 b$

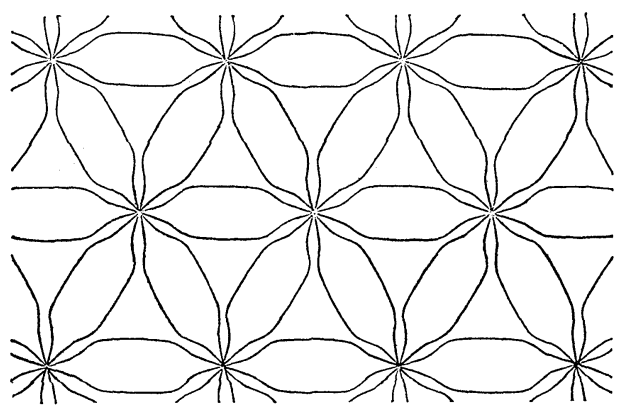

IT $5 \mathrm{~b}$

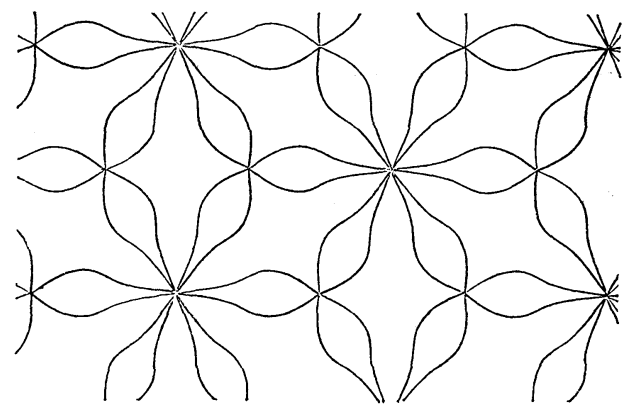

IT $11 b$

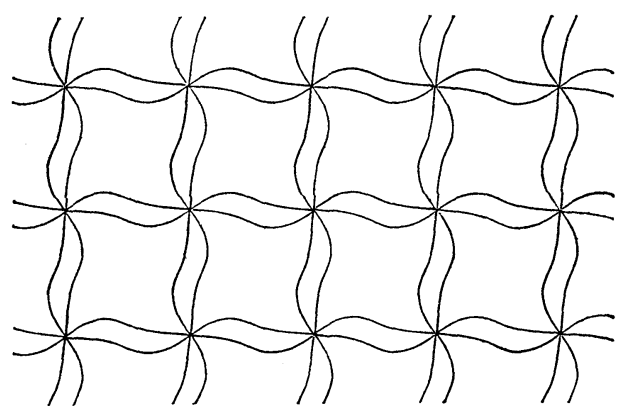

IT $19 \mathrm{~b}$

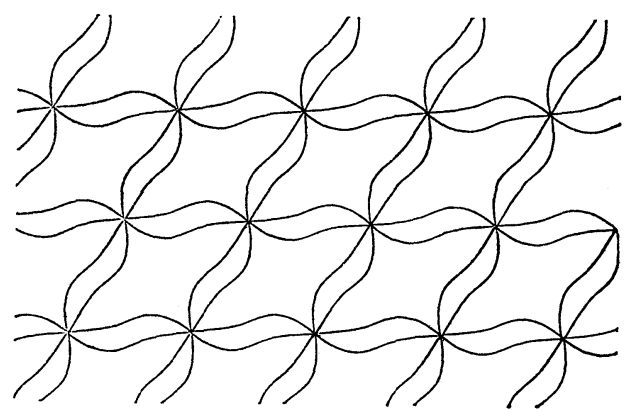

IT $20 \mathrm{~b}$

FiguRE 6 . The fifteen types of isotoxal tilings that include digons. 


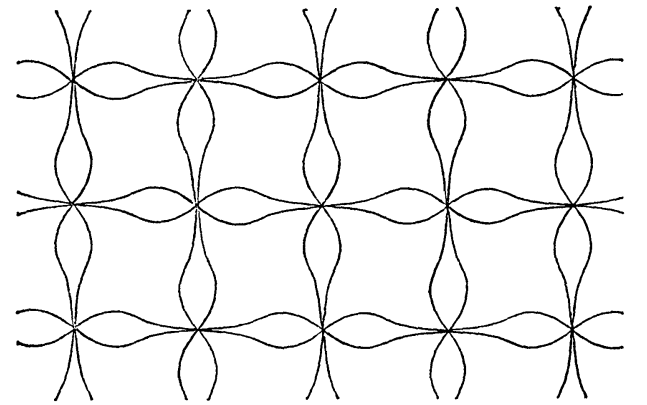

IT $21 \mathrm{~b}$

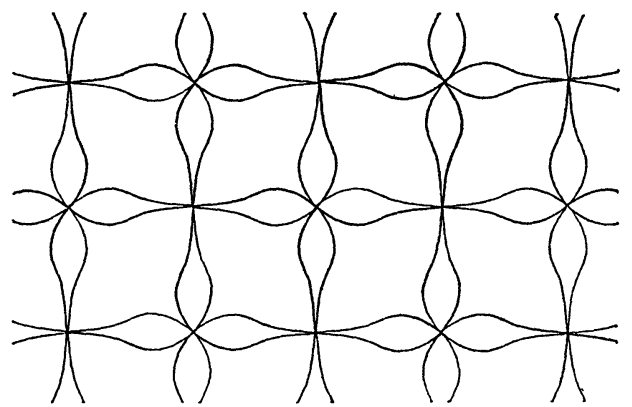

IT $22 \mathrm{~b}$

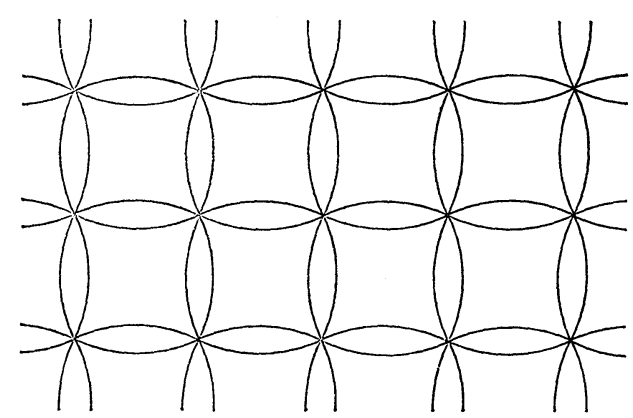

IT $25 \mathrm{~b}$

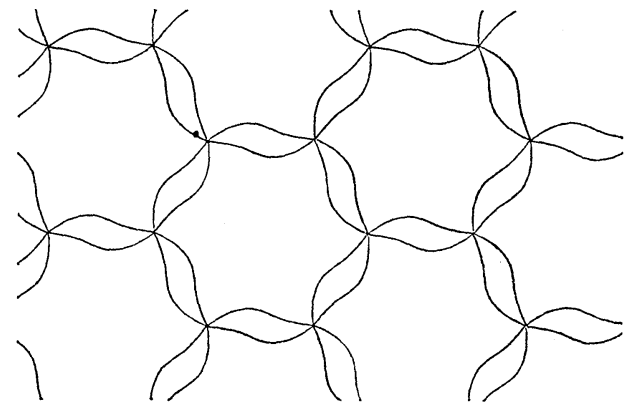

IT $27 \mathrm{~b}$

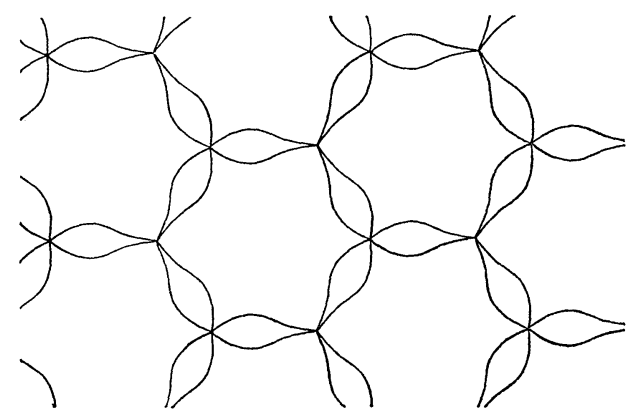

IT $28 \mathrm{~b}$

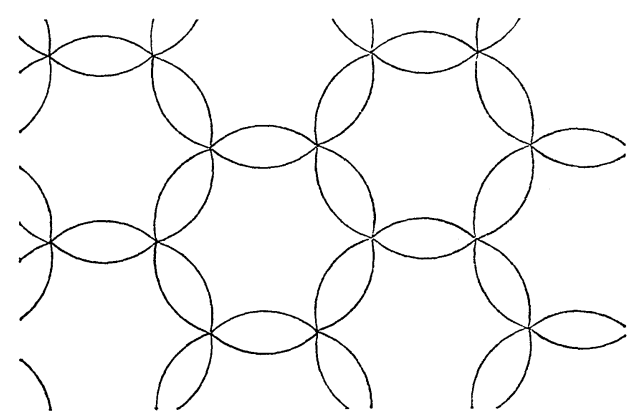

IT $30 \mathrm{~b}$

Figure 6 (continued). 


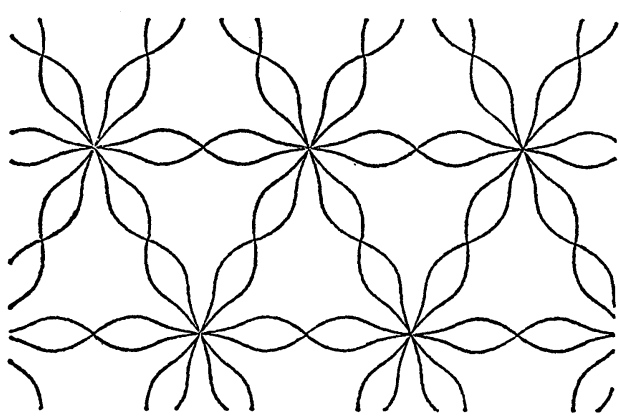

IT $5 \mathrm{db}$

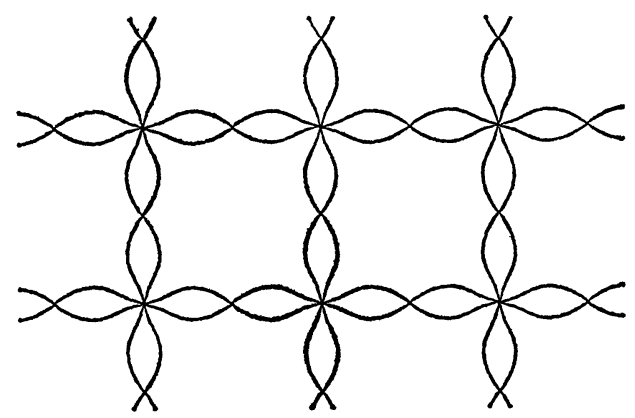

IT $25 \mathrm{db}$

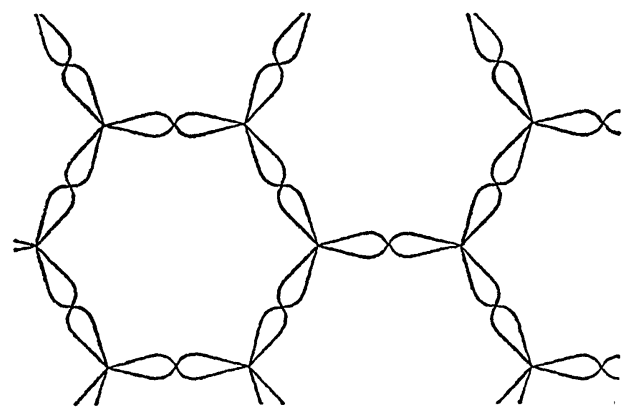

IT $30 \mathrm{db}$

Figure 6 (end).

tiling IT $30 \mathrm{~b}$ with net $\left\langle 2.6 ; 6^{2}\right\rangle$ by bifurcating its edges, a tiling IT 30d with net $\left\langle 12^{2} ; 2.3\right\rangle$ by dividing its edges, and two further tilings IT $30 \mathrm{bd}$ with net $\langle 4.12 ; 2.6\rangle$ and IT $30 \mathrm{db}$ with net $\langle 2.12 ; 4.6\rangle$ by applying both operations consecutively. All these new tilings will be said to be derived from IT 30 , and a full list of derived tilings is given in column (12) of Table I. In fact, as is easily checked, all these new combinatorial types can be realized by shaped tiles even if the original tiling had no such realization.

In all we obtain 15 additional tilings with digons, which are illustrated in Figure 6, and 15 additional tilings with vertices of valence 2. These are not illustrated since they can be simply obtained by inserting an extra vertex at the midpoint of each edge of tilings IT 2 , IT 4, IT 5, IT 8, IT 19, IT 20, IT 23, IT 24, IT 25, IT 27 , IT 29 , IT 30 , IT $5 \mathrm{~b}$, IT $25 \mathrm{~b}$, and IT $30 \mathrm{~b}$.

To complete the proof of Theorem 2 we need only remark that the procedure described above yields all the tilings of the required types. This is a consequence of the following fact, which may be easily verified. Starting with any one of the fourteen possible nets, labelled in the usual manner, we can reduce it to one of the 30 combinatorial types listed in Table I by applying the inverses of 
edge-bifurcation and of edge-division, or of both operations consecutively.

4. In [3] we pointed out that each combinatorial type of isogonal tiling is the dual of a combinatorial type of isohedral tiling, and conversely. This is apparent from the observation that the adjacency symbol of any such tiling is identical with the adjacency symbol of its dual. In a similar way, there exists a theory of duality for isotoxal tilings-we shall first explain how the dual is constructed and then relate this construction to a "dual adjacency symbol".

Let $\mathscr{T}$ be any isotoxal tiling. A topological dual $\mathscr{T}^{*}$ of $\mathscr{T}$ may be constructed by taking an interior point of each tile of $\mathscr{T}$ as a vertex of $\mathscr{T}^{*}$, and joining two such vertices by an edge $\alpha^{*}$ if and only if the corresponding tiles have an edge $\alpha$ in common. The edges $\alpha^{*}$ may be chosen to be any simple curves of finite length subject only to the condition that they be disjoint except possibly at their endpoints. A combinatorial dual is obtained by first constructing a topological dual $\mathscr{T}^{*}$ and then assigning symbols to the
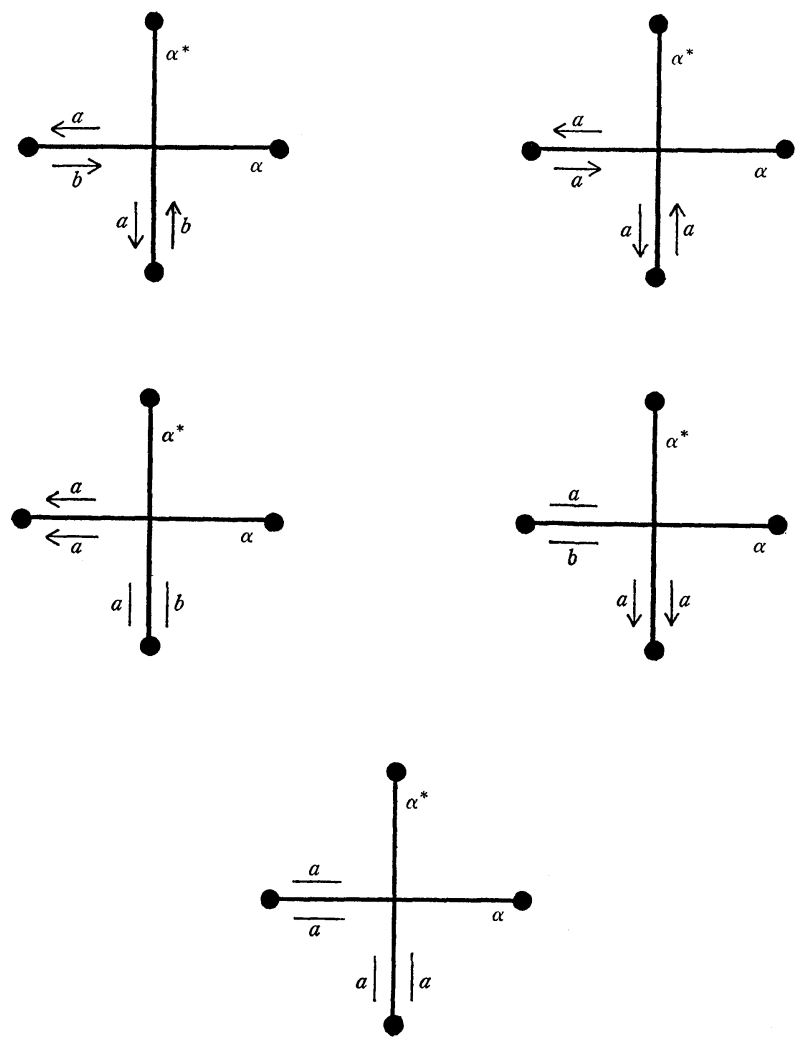

FIGURE 7. Relations of labelled edges in dual isotoxal tilings. 
edges of the tiling according to the following rule (see Figure 7): if $\alpha$ is an edge of type $a^{+} b^{+}, a^{+} a^{+}, a^{+} a^{-}, a b$ or $a a$, then $\alpha^{*}$ must be an edge of type $a^{+} b^{+}, a^{+} a^{+}, a b, a^{+} a^{-}$or $a a$, respectively.

This process, applied to the 30 combinatorial types of Table I, yields the dual types listed in column (10) of the table. There exist 12 dual pairs and the remaining 6 types, marked by an asterisk in the table, are self dual. It must be noted that, contrary to the assertions of some authors, this duality applies only to combinatorial types (and therefore to marked tilings) -it cannot be extended to tilings which are specified by the shapes of the tiles. For example, the dual of tiling IT 21 is IT 24 . The former can be realized only as a marked tiling, whereas the latter corresponds to a "genuine" tiling by shaped tiles.

The justification for the procedure just described (which may, at first sight, appear somewhat arbitrary) depends on the fact that to any isotoxal tiling $\mathscr{T}$ we can assign a dual edge symbol and dual adjacency symbol in the following manner. Instead of assigning symbols $a, b$ to the oriented sides of the tiles of $\mathscr{T}$, we use them for the sensed ends of the edges of $\mathscr{T}$ in precisely the same manner as described in [3]. Then the edges are of five types, namely $a^{+} b^{+}$, $a^{+} a^{+}, a^{+} a^{-}, a b$, and $a a$, which are the dual edge symbols. Here $a^{+} b^{+}$means that the ends of an edge bear the symbol $a, b$ and the superscripts ${ }^{++}$signify that they are sensed in the same direction (clockwise or counterclockwise about their respective vertices). The other four dual edge symbols have the obvious interpretations. To define the dual adjacency symbols we read off, in cyclic order round each of the two vertices at the ends of an edge of $\mathscr{T}$, the symbols assigned to the ends of the edges meeting at those vertices. We use superscripts ${ }^{+},-$or neither to show that the ends are sensed coherently, oppositely, or are not sensed, in the same manner as described in [3]. It is easily verified that the following is true:

If $\mathscr{T}^{*}$ is the dual combinatorial tiling of the isotoxal tiling $\mathscr{T}$, constructed in the manner described above, then $\mathscr{T}^{*}$ is isotoxal and the edge and adjacency symbols of $\mathscr{T}$ are the same as the dual edge and dual adjacency symbols of $\mathscr{T}^{*}$.

Not only does this justify our construction, but it also shows that we might, if we had so wished, have carried out the enumeration of isotoxal tilings using dual symbols. In fact, such a procedure would have been completely equivalent to that described in $\S 2$.

From Figures 3, 4, and 6 we see that the tiles of any isotoxal tiling $\mathscr{T}$ have a nontrivial symmetry group induced by the symmetry group $S(\mathscr{T})$ of $\mathscr{T}$. This observation is relevant to the 


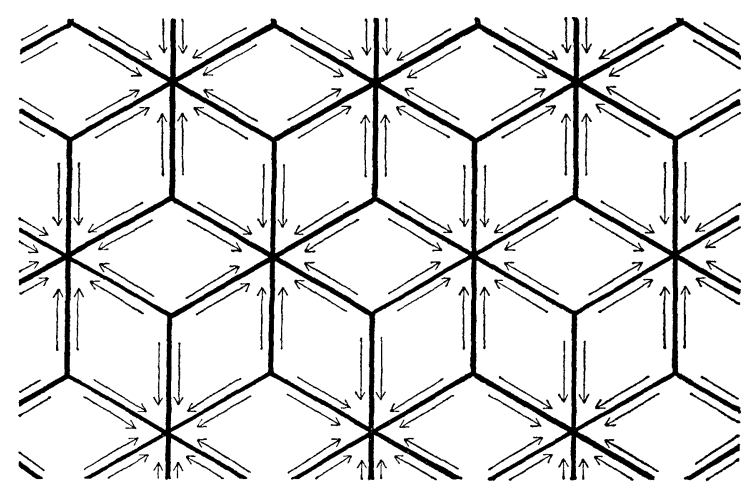

(a) $\mathscr{T}$ of type IT 11.

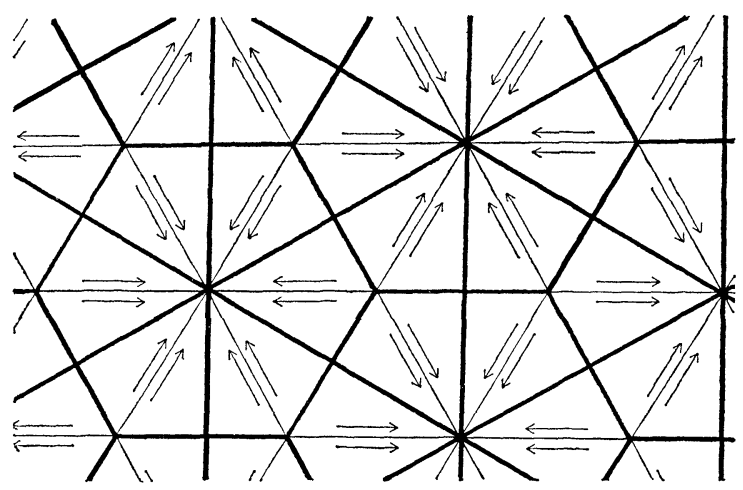

(b) $\mathscr{T}_{c}$ of type IH 32.

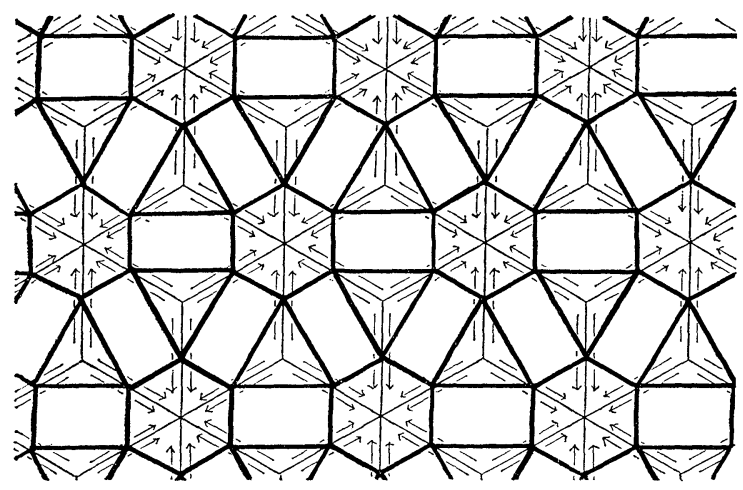

(c) $\mathscr{T}_{a}$ of type IG 32.

Figure 8. Centered tilings. 
following description of two procedures which relate isotoxal to isohedral and isogonal tilings (see Figure 8).

Let $\mathscr{T}$ be a normal isotoxal tiling, which we take in the form of one of the standard nets of Figure 1, with the sides of the tiles labelled in some manner. A new marked tiling $\mathscr{T}_{c}$ is then constructed as follows. The vertices of $\mathscr{T}_{c}$ are defined to be the vertices of $\mathscr{T}$, together with one point in the interior of each tile of $\mathscr{T}$. These points must be selected in such a manner that the marked tiling consisting of $\mathscr{T}$ with each tile marked also by the chosen point has the same combinatorial type as $\mathscr{T}$. Thus we begin by selecting a point $t$ in the interior of the tile $T \in \mathscr{T}$ in such a way that it is left invariant by those operations of $S(\mathscr{T})$ which map $T$ onto itself. The images of $t$ under the operations of $S(\mathscr{T})$ are then also vertices of $\mathscr{T}_{c}$. The edges of $\mathscr{T}_{c}$ are straight line segments joining each vertex of $\mathscr{T}_{c}$ in the interior of a tile $T$ to the vertices of $T$. Clearly each edge of $\mathscr{T}$ lies entirely within one tile of $\mathscr{T}_{c}$, and so we may take these edges, along with their labels, to be markings of $\mathscr{T}_{c}$. The marked tiling $\mathscr{T}_{c}$ thus defined is said to be obtained from $\mathscr{T}$ by tile-centering and it is immediate from the isotoxality of $\mathscr{T}$ and the method of construction that $\mathscr{T}_{c}$ is isohedral. (Figure 8a, in which the arrows indicate the oriented sides labelled $a$, shows the isotoxal tiling $\mathscr{T}$ of type IT 11, and Figure $8 \mathrm{~b}$ the corresponding $\mathscr{T}_{c}$ of type IH 32.)

In the second procedure we again construct a marked tiling, here denoted by $\mathscr{T}_{a}$, from a given labelled isotoxal tiling $\mathscr{T}$. The vertices of $\mathscr{T}_{a}$ consist of one point selected on each edge of $\mathscr{T}$ in such a way that the set of all vertices is invariant under $S(\mathscr{T})$. The edges of $\mathscr{T}_{a}$ are straight line segments joining each vertex $V$ to the four vertices lying on neighboring sides of the two tiles of $\mathscr{T}$ incident with $V$. Each node of $\mathscr{T}_{a}$ divides an edge of $\mathscr{T}$ into two parts, each of which lies entirely within a tile of $\mathscr{T}_{a}$. These half-edges, bearing the labels of the tiling $\mathscr{T}$, are to be interpreted as markings on the tiles of $\mathscr{T}_{a}$. The marked tiling thus defined is said to be obtained from $\mathscr{T}$ by edge-centering. It follows immediately from the isotoxality of $\mathscr{T}$ and the method of construction that $\mathscr{T}_{a}$ is isogonal. (Figure $8 \mathrm{c}$ shows $\mathscr{T}_{a}$ of type IG 32 corresponding to the isotoxal tiling $\mathscr{T}$ of Figure 8a.)

It also follows that $\mathscr{T}_{c}$ and $\mathscr{T}_{a}$ are dual combinatorial types of tiling. Moreover, if $\mathscr{T}_{,} \mathscr{T}^{*}$ are dual isotoxal tilings then $\mathscr{T}_{c}, \mathscr{T}_{c}^{*}$ are isohedral tilings of the same type, and $\mathscr{T}_{a}, \mathscr{T}_{a}^{*}$ are isogonal tilings of the same type. In column (12) of Table I an entry $n$ means that $\mathscr{T}_{c}$ is of type IH $n$ and $\mathscr{T}_{\alpha}$ is of type IG $n$, these numbers referring to the lists of isohedral and isogonal tilings given in [2] and [3]. The processes of tile-centering and edge-centering 
can also be applied, with obvious modifications, to derived isotoxal tilings, that is to say, those bounded isotoxal tilings that contain digons or vertices of valence 2 . In each case it is a simple matter to identify the isohedral tiling $\mathscr{T}_{c}$ and the isogonal tiling $\mathscr{T}_{a}$; however, in this situation the tilings $\mathscr{T}_{c}$ and $\mathscr{T}_{a}$ are not normal since they contain digons or vertices of valence 2 , and therefore have not been listed in the tables of [2] and [3].

We mention the processes of tile-centering and edge-centering because, apart from their intrinsic interest, they can also lead to alternative methods of enumerating isotoxal tilings. It is possible to formulate conditions which characterize those isohedral and isogonal tilings which can be obtained from isotoxal tilings as described. However, the details of this are rather complicated and it is simpler to use the method described in $\S 2$.

\section{REFERENCES}

1. B. N. Delone, Teoriya planigonov, Izv. Akad. Nauk SSSR, Ser. Matem. 23 (1959), $365-386$.

2. B. Grünbaum and G. C. Shephard, The eighty-one types of isohedral tilings in the plane, Math. Proc. Cambridge Phil. Soc., 82 (1977), 177-196.

3. - The ninety-one types of isogonal tilings in the plane, Trans. Amer. Math. Soc., (to appear).

4. - Isohedral, isogonal and isotoxal tilings on the sphere, (to appear).

5. H. Heesch, Über topologisch gleichwertige Kristallbindungen, Zeitschrift für Kristallographie, 84 (1933), 399-407.

6. K. Reinhardt, Über die Zerlegung der Ebene in Polygone, Dissertation, Universität Frankfurt, 1918.

Received August 5, 1976. Research supported by the National Science Foundation through Grant MPS74-07547 A01 and the National Research Council of Canada, Grant A 7071 .

UNIVERSITY OF WASHINGTON

SEATTLE, WA 98195

AND

UNIVERSITY OF EAST NORWICH

NR14 8QJ 


\section{PACIFIC JOURNAL OF MATHEMATICS}

\section{EDITORS}

RICHARD ARENS (Managing Editor)

University of California

Los Angeles, CA 90024

Charles W. Curtis

University of Oregon

Eugene, OR 97403

C. C. MOORE

University of California

Berkeley, CA 94720

\section{J. DugundJI}

Department of Mathematics

University of Southern California

Los Angeles, CA 90007

R. FinN and J. Milgram

Stanford University

Stanford, CA 94305

\section{ASSOCIATE EDITORS}
E. F. BECKENBACH
B. H. NeumanN
F. WOLF
K. YosHIDA

\section{SUPPORTING INSTITUTIONS}

\author{
UNIVERSITY OF BRITISH COLUMBIA \\ CALIFORNIA INSTITUTE OF TECHNOLOGY \\ UNIVERSITY OF CALIFORNIA \\ MONTANA STATE UNIVERSITY \\ UNIVERSITY OF NEVADA, RENO \\ NEW MEXICO STATE UNIVERSITY \\ OREGON STATE UNIVERSITY \\ UNIVERSITY OF OREGON
}

\author{
UNIVERSITY OF SOUTHERN CALIFORNIA \\ STANFORD UNIVERSITY \\ UNIVERSITY OF HAWAII \\ UNIVERSITY OF TOKYO \\ UNIVERSITY OF UTAH \\ WASHINGTON STATE UNIVERSITY \\ UNIVERSITY OF WASHINGTON
}

The Supporting Institutions listed above contribute to the cost of publication of this Journal, but they are not owners or publishers and have no responsibility for its content or policies.

Mathematical papers intended for publication in the Pacific Journal of Mathematics should be in typed form or offset-reproduced, (not dittoed), double spaced with large margins. Please do not use built up fractions in the text of the manuscript. However, you may use them in the displayed equations. Underline Greek letters in red, German in green, and script in blue. The first paragraph or two must be capable of being used separately as a synopsis of the entire paper. Items of the bibliography should not be cited there unless absolutely necessary, in which case they must be identified by author and journal, rather than by item number. Manuscripts, in triplicate, may be sent to any one of the editors. Please classify according to the scheme of Math. Reviews, Index to Vol. 39. All other communications should be addressed to the managing editor, or Elaine Barth, University of California, Los Angeles, California, 90024.

50 reprints to each author are provided free for each article, only if page charges have been substantially paid. Additional copies may be obtained at cost in multiples of 50 .

The Pacific Journal of Mathematics is issued monthly as of January 1966. Regular subscription rate: $\$ 72.00$ a year (6 Vols., 12 issues). Special rate: $\$ 36.00$ a year to individual members of supporting institutions.

Subscriptions, orders for numbers issued in the last three calendar years, and changes of address should be sent to Pacific Journal of Mathematics, 103 Highland Boulevard, Berkeley, California, 94708. Older back numbers obtainable from Kraus Periodicals Co., Route 100, Millwood, NY 10546.

PUBLISHED BY PACIFIC JOURNAL OF MATHEMATICS, A NON-PROFIT CORPORATION

Printed at Kokusai Bunken Insatsusha (International Academic Printing Co., Ltd.). 8-8, 3-chome, Takadanobaba, Shinjuku-ku, Tokyo 160, Japan.

Copyright (C) 1978 by Pacific Journal of Mathematics Manufactured and first issued in Japan 


\section{Pacific Journal of Mathematics}

\section{Vol. 76, No. $2 \quad$ December, 1978}

Stephanie Brewster Brewer Taylor Alexander, Local and global convexity in complete Riemannian manifolds ...........................

Claudi Alsina i Català, On countable products and algebraic convexifications of probabilistic metric spaces ...............................

Joel David Berman and George Grätzer, Uniform representations of

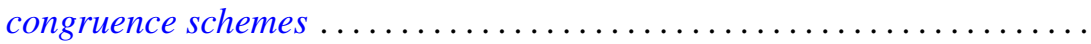

Ajit Kaur Chilana and Kenneth Allen Ross, Spectral synthesis in

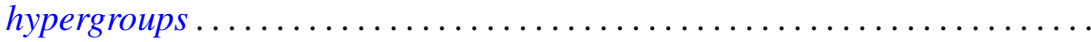

David Mordecai Cohen and Howard Leonard Resnikoff, Hermitian quadratic forms and Hermitian modular forms . .........................

Frank Rimi DeMeyer, Metabelian groups with an irreducible projective

representation of large degree .............................

Robert Ellis, The Furstenberg structure theorem .....................

Heinz W. Engl, Random fixed point theorems for multivalued mappings .......

William Andrew Ettling, On arc length sharpenings ..................

Kent Ralph Fuller and Joel K. Haack, Rings with quivers that are trees........

Kenneth R. Goodearl, Centers of regular self-injective rings ...............

John Gregory, Numerical algorithms for oscillation vectors of second order

differential equations including the Euler-Lagrange equation for

symmetric tridiagonal matrices.

Branko Grünbaum and Geoffrey Shephard, Isotoxal tilings

Myron Stanley Henry and Kenneth Leroy Wiggins, Applications of

approximation theory to differential equations with deviating

arguments

Mark Jungerman, The non-orientable genus of the n-cube .

Robert Richard Kallman, Only trivial Borel measures on $S_{\infty}$ are

quasi-invariant under automorphisms ................

Joyce Longman and Michael Rich, Scalar dependent algebras in the

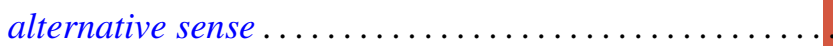

Richard A. Mollin, The Schur group of a field of characteristic zero ........ 471

David Pokrass, Some radical properties of rings with $(a, b, c)=(c, a, b) \ldots 479$

Margaret Shay and Paul Ruel Young, Characterizing the orders changed by

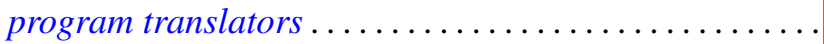

Jerrold Norman Siegel, On the structure of $B_{\infty}(F), F$ a stable space...

Surjeet Singh, (hnp)-rings over which every module admits a basic

submodule...

A. K. Snyder, Universal interpolating sets and the Nevanlinna-Pick property in

Banach spaces of functions...

Jeffrey D. Vaaler, On the metric theory of Diophantine approximation ... 